\title{
The variations of solar magnetic networks over the solar cycle 23
}

\author{
Chong Huang ${ }^{1}$, Yihua Yan ${ }^{1}$, Yuanyong Deng ${ }^{1}$, Yin Zhang ${ }^{1}$, \\ Baolin $\operatorname{Tan}^{1}$ and Gang $\mathbf{L i}^{1,2}$ \\ ${ }^{1}$ Key Laboratory of Solar Activity, National Astronomical Observatories of the Chinese \\ Academy of Sciences, Beijing 100012, China; \\ ${ }^{2}$ Department of Physics and CSPAR, University of Alabama in Huntsville, AL, 35899, USA \\ email: chuang@nao.cas.cn
}

\begin{abstract}
This work analyzed the morphologic properties of magnetic networks during Carrington Rotations (CRs) 1955 to 2091 by applying the watershed algorithm to magnetograms observed by the Michelson Doppler Interferometer on board the Solar and Heliospheric Observatory spacecraft. We found that the magnetic networks are of fractal and the average fractal dimension is $D_{f}=1.253 \pm 0.011$. We also find that both the fractal dimension and the size of magnetic networks are anti-correlated with the solar magnetic activity.
\end{abstract}

Keywords. variation, solar activity, magnetic network

\section{Introduction}

Solar magnetic fields are generated by the motion of conductive plasma in the highly turbulent convective layers. Their emergence manifests the magnetic networks at the solar photosphere. Although research in magnetic networks have undergone tremendous progress, the interaction between magnetic network and magnetic activity is still not well resolved. In this paper, we use the watershed algorithm to identify the magnetic networks for the data of MDI/SOHO over the solar cycle 23, and perform statistic analysis of the cell sizes and the fractal dimension as a function of the solar activity level.

\section{Observations and Results}

The synoptic charts analyzed in this work are obtained by MDI/SOHO during the Carrington Rotation (CR) 1955 to CR 2091. We select a region of CR synoptic charts that situates between $15^{\circ} \mathrm{S}-15^{\circ} \mathrm{N}$ latitude in heliographic coordinates to measure the morphologic properties of the magnetic networks. Since the watershed algorithm depends on the choice of smoothing scale, we apply two methods to deprive the standard smoothing scale. First, chromospheric networks can be easily extracted manually as objective things, so they can be used as commendable indicators to the magnetic networks. We found chromospheric networks can be identified well with watershed algorithm in a smoothing scale of 3.7 pixel $(5.08 \mathrm{Mm})$, and they can match magnetic networks (with the same image process) well. Furthermore, by using the derived smoothing scale of 3.7 pixels with watershed algorithm, we can obtain the average cell size of $20 \mathrm{Mm}$, which is the same size from the FFT spectrum. Thus, we suggest that the smoothing scale of 3.7 pixels can be used as a standard to extract the network. In the following, we therefore use a smoothing scale of $=3.7$ pixels in the watershed method.

At present, the relation between cell size and the activity level is still highly debatable. We investigate the cells over ten years on magnetogram observations, obtaining the change of the mean network cell size for each Carrington Rotation. Over 574,000 cells are 
identified and measured, the results are shown in left panel of Figure 1 where the relation between cell scale size $L$ and the magnetic activity is apparent. This inverse correlation shows that on average a cell's size increases as solar activity decreases, in agreement with earlier works Singh \& Bappu (1981).
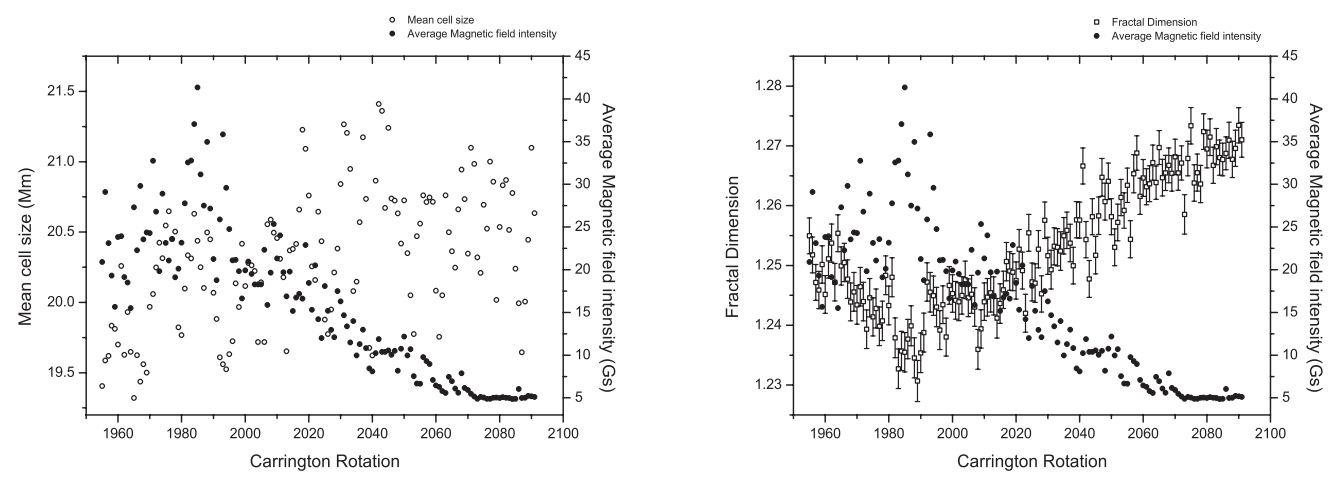

Figure 1. Left: mean cell size (the empty ball) during CR $1955-2091$ from $15^{\circ} \mathrm{S}$ to $15^{\circ} \mathrm{N}$ versus magnetic activity (the filled ball). Right: comparison between fractal dimensions (the empty square) and magnetic activity (the filled ball) during CR $1955-2091$ from $15^{\circ} \mathrm{S}$ to $15^{\circ} \mathrm{N}$. Error bars are \pm 1 standard deviation of the mean.

On the other hand, a fractal analysis can quantify the irregularity of a magnetic network and reveal the intrinsic features of the turbulence on the solar surface. Here we apply a fractal analysis which follows Mandelbrot (1977), Nesme-Ribes et al. (1996) on the magnetic networks obtained from the above watershed algorithm. The fractal dimension $D_{f}$ is defined by the area-perimeter relationship: $P \propto A^{D_{f} / 2}$, where $P$ is the perimeter and $A$ is the area. We acquire the average fractal dimension is $D_{f}=1.253 \pm 0.011$, and examine the relationship between the fractal dimensions of network cell and the solar magnetic activity level. The right panel of Figure 1 plots the fractal dimension $D_{f}$ and magnetic activity for all CRs investigate in this paper. This indicates that the correlation between the fractal dimension of magnetic networks and magnetic activity is stronger than the correlation between the average scale size of cells and magnetic activity.

\section{Conclusion}

The average cell scale size weakly anti-correlates with the solar magnetic activity. The fractal dimension of magnetic network cells also anti-correlates with the activity level, and the correlation is stronger. From the Kolmogorov theory of homogeneous and isotropic turbulence, we suggest that the strong magnetic field may suppress spatially modulated oscillation, reduce the scale of the cell, and compress their boundaries, making the boundaries smoother. More details are shown in Huang et al. (2012).

\section{Acknowledgements}

This work is supported by NSFC Grant No. 10921303, MOST Grant No. 2011CB811401, and the National Major Scientific Equipment R\&D Project ZDYZ2009-3.

\section{References}

Huang, C., Yan, Y. H., Zhang, Y., Tan, B. L., \& Li, G. 2012, ApJ , 759, 106

Mandelbrot, B. B. 1977, The Fractal Geometry of the Nature, W. H. Freeman and co., Newyork Nesme-Ribes, E., Meunier, N. \& Collin, B. 1996, ApJ, 308, 213

Singh, J. \& Bappu, M. K. V. 1981, Solar Phys., 71, 161 